\title{
Therapies for COVID-19: A Disorganized Approach
}

\author{
John Somberga, b
}

Over this past year the world has faced a one in a century pandemic that has disrupted people's lives on a massive scale and the world economy. While vaccines are central to combating the pandemic, alternative therapies will play a part in saving lives and modulating the severity of coronavirus disease 2019 (COVID-19). Previously I have commented on the needless politicization of drug testing, specifically relating to chloroquine and hydroxychloroquine treatment. Unfortunately, we have not progressed in this regard and as a medical community still have political and disjointed approaches to clinical trials in this area.

It would seem appropriate in these critical times to have a well-organized approach to evaluating therapies. The National Institutes of Health (NIH) and the Centers for Disease Control and Prevention (CDC) have the resources or could request from Congress the resources to be able to organize large-scale clinical trials evaluating therapeutic approaches to COVID-19. These clinical studies would logically focus on early therapies; late stages of the illness involve multiple organ symptoms with intense severity making therapy significantly more difficult and very different from initial therapy. Early treatment seems the best period to diminish disease severity. However, the studies on hydroxychloroquine and in ivermectin, two potential early treatments are inadequate in size and mostly with the former drug focus is on hospitalized, advanced disease patients.

We also have conflicting study results with convalescent blood plasma in part because of small trials, varying populations studied and for the most part patients with late disease. Some studies show treatment promise and others do not. Based on conflicting data the Food and Drug Administration (FDA) has revoked the emergency use authorization for the use of convalescent blood plasma for COVID-19 treatment in patients in early stages, restricting use to severely ill, hospitalized patients. Would it not be better to encourage more organized studies and especially large, well powered studies targeting patients with comorbidities putting them at higher risk who early on have mild, possibly treatable disease? The USA has severely curtailed its economy and the activities of its population to reduce infection and reduce the burden on our hospitals and health care system. Unfortunately treatments for the

Manuscript submitted February 7, 2021, accepted February 9, 2021

Published online February 23, 2021

aCardiology \& Pharmacology, Rush University, Chicago, IL 60612, USA. Email: John_Somberg@rush.edu

bEditor-in-Chief, Cardiology Research

doi: https://doi.org/10.14740/cr1237 severely ill patients have been our focus, while letting milder infections run their course till a subset of those infected are hospitalized. Voice should be given to the need for early interventions, the need to design adequately powered studies in the less sick, predominantly outpatients. There lies the greatest public health benefit.

The USA and other countries with the medical science resource needed to plan and formulate a programmatic approach to therapeutics in this area depending on individual researchers and medical research centers developing trials on an ad hoc basis. While this system works over the long haul in times of crisis such as war or pandemic, a far more organized and better planned program is needed.

As an example, I am assisting a small research company developing a nitric oxide treatment for early COVID-19. It is most difficult to find physician groups, or medical centers that provide early diagnosis for COVID-19 and are willing to enroll patients. The vaccine trials have also found identifying volunteers difficult; and companies have had to initiate international trials for recruitment that are more costly and difficult to manage and at times delaying recruitment and study outcomes. Could we not develop a system to register investigators willing to participate in multicenter trials in all stages of the disease? Especially in studies of early COVID-19 treatment we need to involve testing sites to identify patients to be treated in the first $72 \mathrm{hrs}$ of their disease.

Even after a year with vaccination programs underway, we still need more coordination in the research for COVID-19. We all hope the vaccine will end the pandemic, but it may turn out to be like it is with influenza with changing viral variants every so often, requiring modification of the vaccine with still tens of thousands of patients still being infected and needing additional therapeutic options.

\section{Acknowledgments}

None to declare.

\section{Financial Disclosure}

None to declare.

\section{Conflict of Interest}

None to declare. 


\section{Data Availability}

The author declares that data supporting the findings of this study are available within the article. 